\title{
VARIACIÓN MORFOLÓGICA in situ DE Carica papaya L. NATIVA DE MÉXICO
}

\author{
In situ MORPHOLOGICAL VARIATION OF Carica papaya L. NATIVE TO MEXICO
}

\author{
Gregorio Hernández-Salinas ${ }^{1,2}$, Alejandra Soto-Estrada*, Eliseo García-Pérez', \\ Arturo Pérez-Vázquez ${ }^{1}$, Mario Rocandio-Rodríguez ${ }^{3}$ y Leobigildo Córdova-Téllez ${ }^{4}$
}

\begin{abstract}
'Colegio de Postgraduados, Campus Veracruz, Veracruz, México. 'Instituto Tecnológico Superior de Zongolica, Campus Tezonapa, Tepetlitlanapa, Zongolica, Veracruz, México. ${ }^{3}$ Universidad Autónoma de Tamaulipas, Instituto de Ecología Aplicada, Cd. Victoria, Tamaulipas, México. ${ }^{4}$ Colegio de Postgraduados, Campus Montecillo, Texcoco, México.
\end{abstract}

*Autor para correspondencia (alejandras@colpos.mx)

\section{RESUMEN}

En México existen poblaciones silvestres y domesticadas de Carica papaya; sin embargo, los estudios relacionados con su variación in situ han sido limitados en número tanto de ejemplares como de zonas geográficas. El objetivo del presente estudio fue caracterizar in situ la variación morfológica de ejemplares de $C$. papaya nativos de México. Se evaluaron 12 características morfológicas (seis cuantitativas y seis cualitativas) de frutos recolectados de 222 ejemplares de papayos nativos provenientes de 10 estados de las vertientes del Pacífico y Golfo de México. Se realizaron análisis de varianza, de componentes principales (ACP) y de agrupamiento jerárquico con distancias euclidianas. De acuerdo con el análisis de varianza, todas las características morfológicas entre recolectas presentaron diferencias significativas $(P \leq 0.01)$. El ACP reveló que las características de mayor contribución a la variación entre los ejemplares analizados fueron volumen comestible, diámetro de cavidad central, longitud, diámetro, grosor de pulpa, forma del extremo peduncular y forma del fruto. El análisis de conglomerados formó tres grupos fenotípicos estadísticamente diferentes $(P \leq 0.01)$ y detectó continuidad con base en dimensiones y forma del fruto. Ambos análisis multivariados diferenciaron entre tipos silvestres y domesticados recolectados en huertos familiares. La alta variabilidad morfológica entre $C$. papaya es apropiada para establecer un programa nacional de conservación de germoplasma de genotipos con disimilitud genética para usarse en programas de mejoramiento genético.

Palabras clave: Carica papaya, poblaciones silvestres, descriptores morfológicos, México.

\section{SUMMARY}

In Mexico there are wild and domesticated populations of Carica papaya; however, studies related to its variation in situ have been limited in number of both individuals and geographic areas. The objective of this study was to characterize in situ the morphological variation of $C$. papaya specimens native to Mexico. Twelve morphological characteristics (six quantitative and six qualitative) of fruits collected from 222 specimens of $C$. papaya from 10 states of the slopes of the Pacific and the Gulf of Mexico were evaluated. Analysis of variance, principal component (PCA) and hierarchical grouping with euclidean distances were performed. According to the analysis of variance, all the morphological characteristics between accessions showed significant differences $(P \leq 0.01)$. The PCA revealed that the characteristics of greater contribution to the variation among the evaluated specimens were edible volume, diameter of the central cavity, length, diameter, thickness of pulp, shape of the peduncular end and fruit shape. The cluster analysis formed three statistically different phenotypic groups $(P \leq 0.01)$ and detected continuity based on the dimensions and shape of the fruit. Both multivariate analyses showed differences between the wild and domesticated types collected in homegardens. The high morphological variability among $C$. papaya is appropriate to establish a national germplasm conservation program of genotypes with genetic dissimilarity to be used in breeding programs.

Index words: Carica papaya, wild populations, morphological descriptors, Mexico.

\section{INTRODUCCIÓN}

La familia Caricaceae consta de seis géneros y 35 especies (Fuentes y Santamaría, 2014); de éstas, Carica papaya $L$. es la especie más importante por el valor comercial y volumen de ventas del fruto Carica papaya, es una especie originaria del norte de Centroamérica y sur de México, donde existen poblaciones cultivadas y silvestres (Fuentes y Santamaría, 2014; Manshardt y Zee, 1994); muestra alta variación morfológica en características como forma, tamaño, color de epidermis, sabor, sólidos solubles totales en el fruto y porte de la planta (Singh y Kumar, 2010). La caracterización morfo-agronómica es un método tradicional y eficiente que se utiliza ampliamente para conocer la diversidad morfológica de las poblaciones (Castellen et al., 2007).

Debido al predominio actual de variedades mejoradas, las poblaciones nativas cultivadas como Cera Amarilla, Mamey, Zapote y Coco han dejado de tener importancia comercial, por lo que su tamaño poblacional se ha reducido, encontrándose solamente en huertos familiares tradicionales (Ávila et al., 2013, Com. Pers.)' ${ }^{1}$, donde se

TÁvila R. C., G. Hernández S., E. García P., E. Hernández C., F. Palemón A., J. L. Hernández H. y J. Jobo H. (2013) Colecta dirigida de los recursos fitogenéticos de Carica papaya L. In: Resúmenes Ejecutivos Ejercicio Fiscal 2010. Sistema Nacional de Recursos Fitogenéticos para la Alimentación y la Agricultura. Servicio Nacional de Inspección y Certificación de Semilla. Chapingo Estado de México. pp:121-122. https://www.gob.mx/cms/ uploads/attachment/file/232379/Resumenes_ejecutivos_ ejercicio_fiscal_2010.pdf (Agosto 2017). 
utilizan básicamente para autoconsumo (Caballero, 1992) y los pobladores conservan el germoplasma, que es un reservorio de genes valiosos para los fitomejoradores.

Existen estudios que se han realizado para caracterizar la variabilidad de C. papaya en variedades tradicionales locales (Aikpokpodion, 2012; Asudi et al., 2010), variedades mejoradas (Singh y Kumar 2010) y ambas (Sompak et al., 2014), las cuales son de interés comercial. Coppens d'Eeckenbrugge et al. (2007), además de las variedades anteriores, incluyeron poblaciones nativas. Aunque dichos estudios contemplan un número reducido de poblaciones, éstos han contribuido a la planificación de estrategias de manejo, mejoramiento genético, conservación en los bancos de germoplasma y utilización sostenible de la diversidad (Ara et al., 2016).

Existe investigación que involucra a poblaciones de C. papaya nativas de México; ésta se ha realizado predominantemente en el sur de los estados de Yucatán y Baja California Sur. Al respecto, Fuentes y Santamaría (2014) reportaron que las plantas femeninas de las poblaciones silvestres de Yucatán miden $5 \mathrm{~m}$ de altura y producen en promedio 70 frutos con peso de 20 a $35 \mathrm{~g}$. Soriano-Melgar et al. (2016), al evaluar 81 recolectas de papayos domesticados de Baja California Sur encontraron frutos de hasta $1.6 \mathrm{~kg}$. Lo anterior evidencia que la diversidad morfológica in situ de poblaciones de papayo nativas de México está poco explorada y caracterizada en regiones específicas; por ello, existe la necesidad de estudiar la variación existente de C. papaya nativa en diferentes zonas agroecológicas de México.

Las poblaciones nativas de C. papaya de valor comercial que aún se conservan en México y se han adaptado a condiciones edafoclimáticas diversas, son de interés para ciertos agricultores, y las características fenotípicas de sus frutos son las de mayor importancia para los mercados consumidores (Wall y Tripathi, 2014). En México, la riqueza genética nativa se está aprovechando para generar variedades nuevas de papayo con alta productividad y plantas de porte bajo (Vázquez et al., 2014). El conocimiento de la variabilidad genética en papaya es imprescindible en el estudio de la estructura genética de las poblaciones nativas para proponer estrategias de conservación y aprovechamiento (Allendorf et al., 2012). En este contexto, el objetivo del presente estudio fue caracterizar in situ la variación morfológica, con énfasis en frutos, de 222 ejemplares de C. papaya nativos de México.

\section{MATERIALES Y MÉTODOS}

\section{Área de estudio y material biológico}

Durante el periodo de 2009 a 2013 se recolectaron frutos de 222 ejemplares de papayo nativos de 10 estados de las vertientes del Pacífico (Baja California Sur, Guerrero, Oaxaca y Chiapas) y del Golfo de México (Hidalgo, San Luis Potosí, Tamaulipas, Veracruz, Tabasco y Campeche). De cada planta se cosecharon de tres a seis frutos en madurez fisiológica. Al momento de la recolecta de frutos se registró la presencia de ramificación en la planta. Los frutos se guardaron en bolsas de papel, se etiquetaron con los datos de pasaporte correspondientes y se utilizaron para la caracterización.

\section{Variables morfológicas}

Las variables cuantitativas que se midieron en el fruto fueron longitud $(\mathrm{cm})$, diámetro $(\mathrm{cm})$, relación longitud/ diámetro, grosor de pulpa $(\mathrm{cm})$, diámetro de la cavidad central $(\mathrm{cm})$ y volumen comestible $\left(\mathrm{cm}^{3}\right)$, mientras que las variables cualitativas incluyeron forma del fruto, forma del extremo peduncular, forma del extremo distal, prominencia de aristas, forma de la cavidad central y color de la pulpa. Adicionalmente se registró la ramificación de la planta en campo. Las variables se evaluaron de acuerdo con los descriptores propuestos por la UPOV (2008) para C. papaya, excepto el volumen comestible, propuesto por Aikpokpodion (2012), la forma del fruto propuesta en el catálogo de Dantas et al. (2000) y el color de pulpa de acuerdo con las tablas de colores de Munsell Color (Firm) (1977).

\section{Análisis estadístico}

Con los datos de las variables se realizó un análisis de varianza mediante el procedimiento GLM de SAS V.9.1 (SAS Institute, 2004). Posteriormente, se eliminaron variables de pares con coeficiente de correlación menor a 0.5, y con bajo aporte a la variación; con las variables restantes, se obtuvieron los valores y vectores propios mediante la matriz de correlaciones (Rawlings et al., 1988), y con la gráfica de Gabriel (1971) se visualizaron las correlaciones entre variables y su aporte a la variación global (Pla, 1986).

Con las variables seleccionadas se realizó un análisis de componentes principales (ACP), construido con la matriz de correlaciones, la cual se analizó con el método no paramétrico de agrupación Modeclus para estimar el 
número adecuado de agrupaciones y éstas distribuirlas en una gráfica biplot (Gabriel, 1971). Para las agrupaciones se efectuó un análisis de conglomerados con datos estandarizados, usando distancias euclidianas y el método de ligamiento promedio (UPGMA). El punto de corte de este análisis se definió con el estadístico de Pseudo F. Se aplicó una prueba de medias de Tukey $(P \leq 0.05)$ sobre los grupos de C. papaya obtenidos con el dendrograma con el paquete estadístico SAS V.9.1 (SAS Institute, 2004).

\section{RESULTADOS Y DISCUSIÓN}

\section{Variación morfológica}

El análisis de varianza detectó diferencias significativas $(P \leq 0.01)$ en todas las variables morfológicas entre los ejemplares evaluados (datos no mostrados), lo que demuestra la existencia de variación entre ellos. La ramificación de planta y siete de las características del fruto presentaron CV menor de $20 \%$, lo que sugiere poca variación aleatoria de estas características. Da Silva et al. (2008) señalaron que un CV con valores de 20 a $40 \%$ y mayores de $40 \%$ se consideran altos y muy altos, respectivamente. En el presente estudio, la forma de la cavidad central, diámetro de la cavidad central y prominencia de aristas presentaron CV altos (26.69, 20.35 y $21.55 \%$, respectivamente). Para el volumen comestible y forma del fruto, los CV fueron muy altos (66.59 y 50.02, respectivamente) y se deben a la variación amplia de estas dos variables entre los ejemplares por factores aleatorios (Figura 1). Dentro de las características consideradas como de mayor variación, el diámetro de la cavidad central y el volumen comestible coinciden con lo que reportaron Aikpokpodion (2012) y Oliveira et al. (2010), quienes señalaron alta variabilidad entre genotipos de papayo. Ara et al. (2016) indicaron que los valores de CV altos se deben a la variación inter-poblacional, en tanto que Oliveira et al. (2010) los atribuyen a la variación intra-poblacional.

\section{Estructuración de la variación}

Los tres primeros compontes principales (CP) explicaron el $77.4 \%$ de la variación total entre los ejemplares estudiados (Cuadro 1). Las características asociadas al CP1 fueron longitud, diámetro, grosor de pulpa, volumen comestible y diámetro de la cavidad central del fruto, mientras que el extremo peduncular y la forma del fruto se relacionaron con el CP2. En el CP3, sólo la prominencia de aristas del fruto aportó significativamente a la variación (Cuadro 1). En esta investigación las características anteriores contribuyeron mayormente a la variación de $C$. papaya nativa de México y coinciden con las registradas

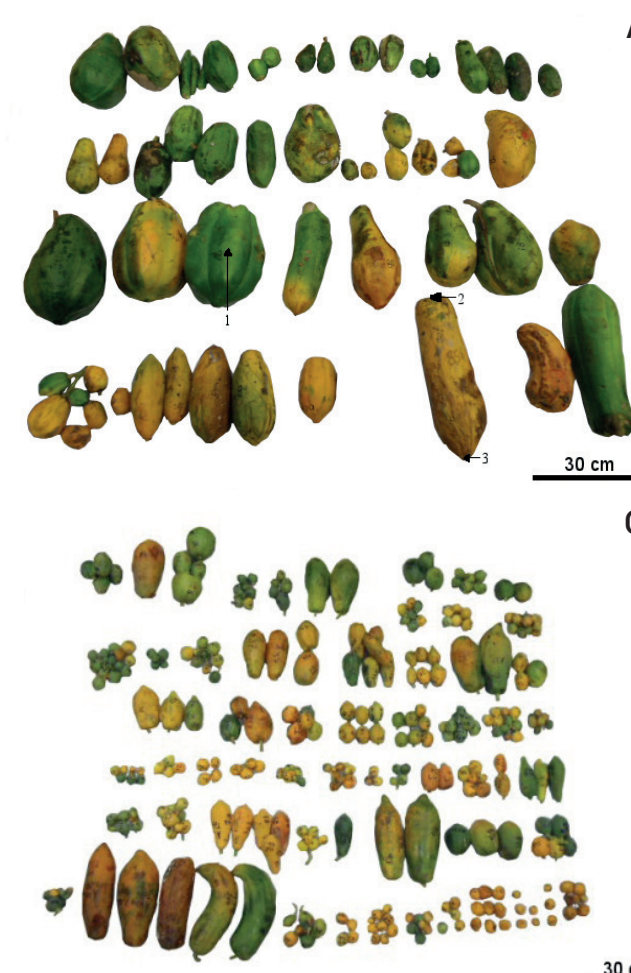

A)

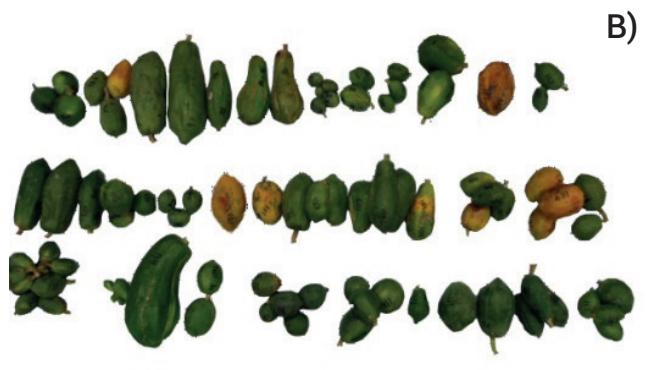

B)

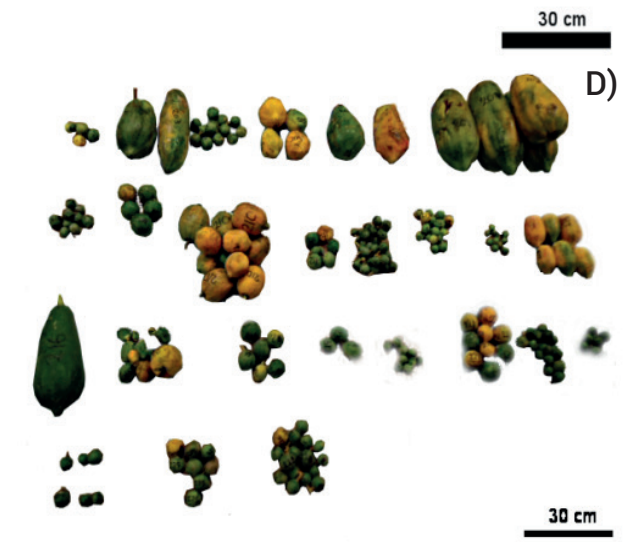

Figura 1. Diversidad morfológica de frutos de Carica papaya recolectados en cuatro estados de México. A) Guerrero, B) Oaxaca, C) Tabasco y D) Campeche. 
Cuadro 1. Vectores propios, valores propios y proporción de la variación explicada de los tres primeros CP de 10 características morfológicas de C. papaya nativa de México.

\begin{tabular}{lccc}
\hline Característica & CP1 & CP2 & CP3 \\
\hline Longitud del fruto $(\mathrm{cm})$ & 0.424 & 0.034 & -0.199 \\
Diámetro del fruto $(\mathrm{cm})$ & 0.405 & -0.228 & 0.055 \\
Grosor de pulpa $(\mathrm{cm})$ & 0.395 & -0.014 & -0.030 \\
Volumen comestible del fruto $\left(\mathrm{cm}^{3}\right)$ & 0.390 & -0.040 & -0.253 \\
Diámetro de la cavidad central $(\mathrm{cm})$ & 0.326 & -0.363 & 0.196 \\
Relación longitud/diámetro & 0.284 & 0.416 & -0.284 \\
Forma del fruto & 0.239 & 0.471 & -0.034 \\
Forma del extremo peduncular & -0.117 & 0.533 & 0.306 \\
Prominencia de aristas & 0.208 & -0.115 & 0.724 \\
Forma de la cavidad central & 0.211 & 0.345 & 0.390 \\
Valor propio & 5.1 & 1.7 & 0.9 \\
Variación explicada (\%) & 51.3 & 16.8 & 9.3 \\
Variación acumulada (\%) & 51.3 & 68.1 & 77.4 \\
\hline
\end{tabular}

en estudios similares, excepto la variable prominencia de aristas del fruto (Aikpokpodion, 2012; Asudi et al., 2010; Oliveira et al., 2012).

Con base en las variables cuantitativas del fruto, los ejemplares se dispersaron gradualmente en el CP1 (Figura 2). Manshardt (2014) señaló que el tamaño, la cavidad ovárica y el color de pulpa han sido las estructuras más favorecidas durante el proceso de domesticación. Así, al cambiar los hábitos de consumo, se fueron prefiriendo los frutos con peso superior a $1 \mathrm{~kg}$ y paralelamente se promovió el hermafroditismo. Este fenómeno se confirmó durante las recolectas del germoplasma de papayo nativo, al observar poblaciones trioicas como las del tipo Mamey (recolecta 87 de Guerrero) con frutos de $43 \mathrm{~cm}$ de longitud y volumen comestible de $5,660.1 \mathrm{~cm}^{3}$.

De acuerdo con el análisis de agrupación Modeclus, a un radio en la hiper-esfera de exploración $\mathrm{R}=0.7$ en el plano de los dos primeros componentes principales, los ejemplares se dispersaron y formaron 11 grupos, distintos fenotípicamente entre ellos.

El Grupo 1 integró 109 ejemplares silvestres que presentaron frutos de dimensiones pequeñas en longitud, diámetro, relación longitud/diámetro, grosor de pulpa, diámetro de cavidad central y volumen comestible de $6.7 \mathrm{~cm}, 4.6 \mathrm{~cm}, 1.5 \mathrm{~cm}, 0.7 \mathrm{~cm}, 3.4 \mathrm{~cm}$ y $31.3 \mathrm{~cm}^{3}$, respectivamente. Cualitativamente, los frutos mostraron forma redonda, cavidad ovárica redonda y aristas pronunciadas ligeramente; además, estos ejemplares silvestres pertenecen exclusivamente a poblaciones dioicas; es decir, las plantas hermafroditas están ausentes, lo cual también han documentado Chávez-Pesqueira y Núñez-Farfán (2016) en México. De las 109 recolectas del Grupo 1,101 proceden de la vertiente del Golfo de México y ocho del Pacífico.

Todos los ejemplares presentaron frutos con dimensiones similares; sin embargo, el CP2 ubicó a los del Pacífico en el Cuadrante II porque presentaron variación en la forma del fruto, la cual fue redonda, oval y elíptica. Las ocho recolectas del Pacífico evidencian que las poblaciones silvestres se encuentran raramente en esta vertiente, ya que la mayoría presenta frutos de tamaño intermedio a grande (Figura 2); esto indica, probablemente, que las barreras montañosas de la Sierra de Juárez y Sierra Madre del Sur han evitado la dispersión de poblaciones externas a ese lugar (Chávez-Pesqueira y Núñez-Farfán, 2016). La pérdida de este germoplasma silvestre también se debe a la destrucción de su hábitat para fines agropecuarios, como se observó directamente durante la recolección de los frutos. La escasez de este material también podría asociarse a una erosión genética baja de la especie (IBPGR, 1986). El Grupo 1 no concentró ejemplares de papayo de Baja California Sur; ésto podría atribuirse a que, en dicho estado, las poblaciones existentes fueron introducidas, pues no se tienen registros de poblaciones silvestres (Badillo, 1971; Soriano-Melgar et al., 2016).

En la parte central del plano (Figura 2) se ubicó el Grupo 2 con 46 ejemplares. Los frutos mostraron dimensiones intermedias en las variables cuantitativas; los valores promedio fueron $13.9 \mathrm{~cm}$ de longitud, $8.0 \mathrm{~cm}$ de diámetro, 


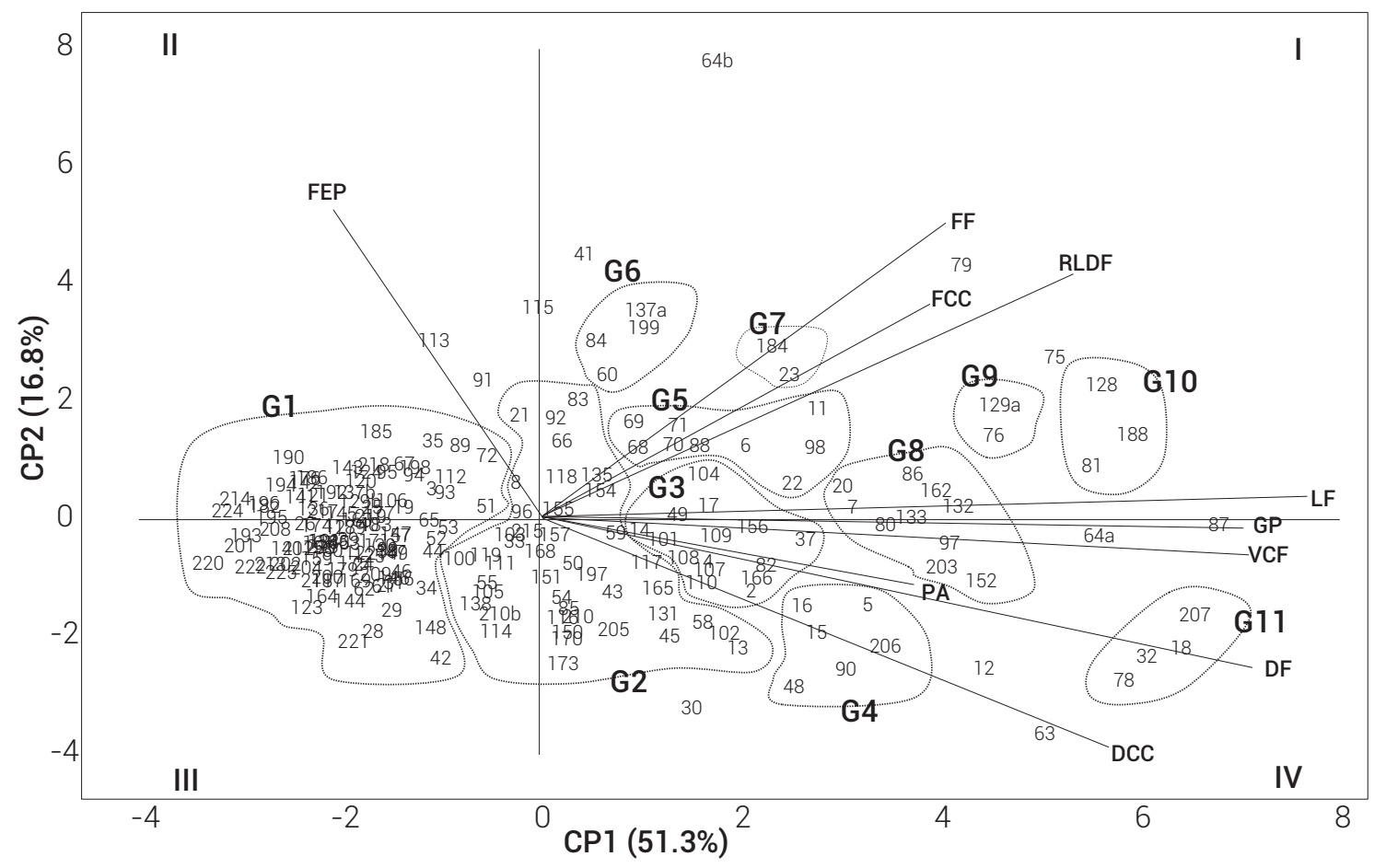

Figura 2. Biplot de 222 ejemplares de Carica papaya elaborado con los dos primeros CP de 10 características morfológicas. Para los acrónimos de las características ver Cuadro 2.

1.8 en la relación longitud/diámetro, $1.5 \mathrm{~cm}$ de grosor de pulpa, $5.1 \mathrm{~cm}$ de diámetro de cavidad central y $293.7 \mathrm{~cm}^{3}$ de volumen comestible. Los frutos tuvieron forma elíptica y cavidad ovárica angular. Este grupo incluyó papayos tanto de tipos silvestres como de domesticados presentes en huertos familiares, con excepción de las recolectas 92 de Guerrero, 105 y 119 de Oaxaca, que se localizaron en hábitat silvestre. Resalta también la recolecta 13 de Baja California Sur que tiene similitud fenotípica con las silvestres de huertos familiares del Grupo 2, aunque ésta es un genotipo domesticado.

El Grupo 3 concentró 15 recolectas de papayo que presentaron frutos con longitud de $19.9 \mathrm{~cm}$, diámetro de $9.8 \mathrm{~cm}$, volumen comestible de $694.1 \mathrm{~cm}^{3}$, de forma elíptica y extremo peduncular hundido. El Grupo 4 integró seis ejemplares con una longitud promedio de $24.6 \mathrm{~cm}$, diámetro de $13.3 \mathrm{~cm}$, volumen comestible de 1203.5 $\mathrm{cm}^{3}$, forma globular y extremo peduncular hundido en el fruto. Se podría inferir que los ejemplares de ambos grupos son domesticados, ya que se observaron plantas hermafroditas durante la recolecta, además de diferentes colores de pulpa como el rojo, donde destaca la recolecta 206 de Campeche (tipo Mamey). Todos los ejemplares se localizaron en huertos familiares.

El Grupo 5 presentó nueve recolectas de C. papaya. El tamaño y volumen comestible del fruto fueron muy similares a los del Grupo 4, pero se diferenciaron por la forma de pera y extremo peduncular achatada del fruto. Dentro de este grupo se encontró la recolecta 22 de Veracruz (tipo Mamey). El Grupo 6 reunió cuatro recolectas; las dimensiones y volumen comestible del fruto fueron similares a las del Grupo 4, distinguiéndose de éste por la forma de pera y extremo peduncular puntiagudo. Los ejemplares de ambos grupos, así como los del 3 y 4 podrían ser domesticados, debido a que se asociaron con la presencia de plantas hermafroditas y varios colores de pulpa como el rojo (Manshardt, 2014).

El Grupo 7 concentró dos ejemplares de huertos familiares; los frutos mostraron similitud en dimensiones y volumen comestible con aquellos del Grupo 3, pero diferente por ostentar frutos en forma de clava y extremo peduncular redondo. La recolecta 23 de Veracruz (Mamey) destacó por ser hermafrodita con frutos de pulpa roja, característica que permite su comercialización regional.

En la parte final del plano del CP1 se posicionaron el Grupo 8 con 10 recolectas, Grupo 9 con dos, Grupo 10 con tres y el Grupo 11 con cuatro. Los frutos presentaron mayores dimensiones en longitud, diámetro, grosor de pulpa, diámetro de cavidad central y volumen comestible que en el resto de los grupos, incluyendo las variedades Zapote, Mamey y Cera Amarilla, que presentan variación en sexos de la planta, formas y colores de pulpa del fruto. 
En estos grupos se posicionaron aquellas recolectas con un grado de domesticación más avanzado; además, se observaron plantas hermafroditas. Los frutos se recolectaron en huertos familiares y se comercializan local o regionalmente, sobre todo los de Cera Amarilla, Mamey y Zapote que dominaron el mercado nacional hasta el año 1990 (Soriano-Melgar et al., 2016) como consecuencia de la introducción de la variedad Maradol a México en 1978.

Los ejemplares de los Grupos 3 al 11 que se encontraron en huertos familiares se distribuyeron ampliamente en los Cuadrantes I y IV (Figura 2), ya que mostraron gran dispersión morfológica entre sí. Estas recolectas ubicadas en esos cuadrantes podrían considerarse como germoplasma del complejo papayo de acuerdo con sus dimensiones, formas del fruto y procedencias diversas. Posiblemente dicho germoplasma nativo ha tenido un manejo de selección mínimo; por tanto, la variación que existe pudiera deberse a los distintos usos locales que se remontan desde la época prehispánica (Manshardt y Zee, 1994) hasta la actualidad (Jiménez, 2011).

La forma del fruto también fue determinante en la discriminación entre recolectas. Segun Nakasone y Paull (1998), los agricultores seleccionan esta característica conforme a las preferencias de los mercados consumidores de papaya. Moore (2014) afirmó que los tipos sexuales están determinados genéticamente, aunque el ambiente también modifica la expresión sexual y al tipo de flor que originará formas de fruto diferentes. Plantas con flores femeninas producen formas esféricas a ovoides, y las hermafroditas producen frutos de forma piriforme o alargada con ciertas variaciones, dependiendo de la variedad y de otros factores (Nakasone y Paull, 1998). Los ejemplares de los Grupos 4, 5, 6, 7, 8, 9, 10 y 11 tuvieron valores de relación longitud/diámetro del fruto superiores a 2.0; este valor está asociado con frutos alargados (Chan y Ooi, 1975).

La variación morfológica amplia detectada en este estudio resalta la importancia de conservar in situ o ex situ germoplasma de papayo nativo. También se evidenció que el proceso de selección y mejoramiento genético consciente $\mathrm{o}$ inconsciente por los pobladores de las diferentes regiones ha cambiado la estructura genética de las poblaciones debido a características fenotípicas de interés centradas en el fruto; por tanto, los propietarios de los huertos deben conservar este germoplasma, pero con medidas de manejo a corto plazo y de esta manera contribuir a la reducción de los cambios genéticos desfavorables a largo plazo (Allendorf et al., 2012).

La amplia diversidad morfológica se debe tanto a la variación inter como a la intra poblacional; además, se asocia con la polinización cruzada, recombinación meiótica y mutación, seguida por una selección intensiva de genotipos en comunidades rurales aisladas y en constante intercambio de material genético (Asudi et al., 2010). El papayo es un cultivo de polinización cruzada en el que el hábito de la floración y fructificación varía, lo que genera una gran diversidad en forma, tamaño, calidad, sabor y color del fruto (Singh y Kumar, 2010). La polinización cruzada ha causado cambios morfológicos en cada población, lo que causa pérdida de identidad en las variedades de papayo y posiblemente la formación de nuevos genotipos (Oliveira et al., 2012).

Los resultados de este estudio apoyan la teoría de que el sur de México es centro de origen de C. papaya (Badillo, 1971; Fuentes y Santamaría, 2014) por la amplia diversidad encontrada, y probablemente centro de domesticación (Fuentes y Santamaría, 2014; Manshardt y Zee, 1994).

\section{Relaciones entre grupos de papayos}

Los agrupamientos de los ejemplares de C. papaya presentaron continuidad en el análisis de conglomerados, principalmente por dimensiones y formas del fruto. Se definieron, de acuerdo con la Pseudo $F$, tres grupos fenotípicos a una distancia euclidiana de 0.95 unidades (Figura 3). Todas las características presentaron diferencias significativas $(P \leq 0.01)$ entre grupos (Cuadro 2).

\section{Cuadro 2. Medias de las características morfológicas del fruto de $C$. papaya nativa de México, en grupos formados por} el dendrograma.

\begin{tabular}{lccccccccccc}
\hline Grupo & $\begin{array}{l}\text { Número de } \\
\text { ejemplares }\end{array}$ & LF $(\mathrm{cm})$ & DF $(\mathrm{cm})$ & RLDF & GP $(\mathrm{cm})$ & DCC $(\mathrm{cm})$ & VCF $\left(\mathrm{cm}^{3}\right)$ & FF & FEP & PA & FCC \\
\hline 1 & 111 & $21.0 \mathrm{a}$ & $9.8 \mathrm{a}$ & $2.1 \mathrm{~b}$ & $2.1 \mathrm{~b}$ & $5.8 \mathrm{a}$ & $1034.6 \mathrm{a}$ & $8.1 \mathrm{~b}$ & $1.5 \mathrm{~b}$ & $1.6 \mathrm{ab}$ & $2.2 \mathrm{~b}$ \\
2 & 3 & $10.5 \mathrm{~b}$ & $4.0 \mathrm{~b}$ & $3.4 \mathrm{a}$ & $3.2 \mathrm{a}$ & $2.0 \mathrm{~b}$ & $77.2 \mathrm{~b}$ & $14.7 \mathrm{a}$ & $3.7 \mathrm{a}$ & $2.0 \mathrm{a}$ & $3.3 \mathrm{a}$ \\
3 & 108 & $6.6 \mathrm{~b}$ & $4.5 \mathrm{~b}$ & $1.5 \mathrm{c}$ & $0.7 \mathrm{c}$ & $3.4 \mathrm{~b}$ & $30.5 \mathrm{~b}$ & $3.5 \mathrm{~b}$ & $1.9 \mathrm{~b}$ & $1.1 \mathrm{~b}$ & $1.3 \mathrm{c}$ \\
\hline
\end{tabular}

Medias con letras iguales en cada columna no son estadísticamente diferentes (Tukey, 0.01). LF: longitud de fruto; DF: diámetro de fruto; RLDF: relación longitud/diámetro; GP. grosor de pulpa; DCC: diámetro de cavidad central; VCF: volumen comestible; FF: forma de fruto; FEP. forma del extremo peduncular; PA: prominencia de aristas; FCC: forma de la cavidad central. 


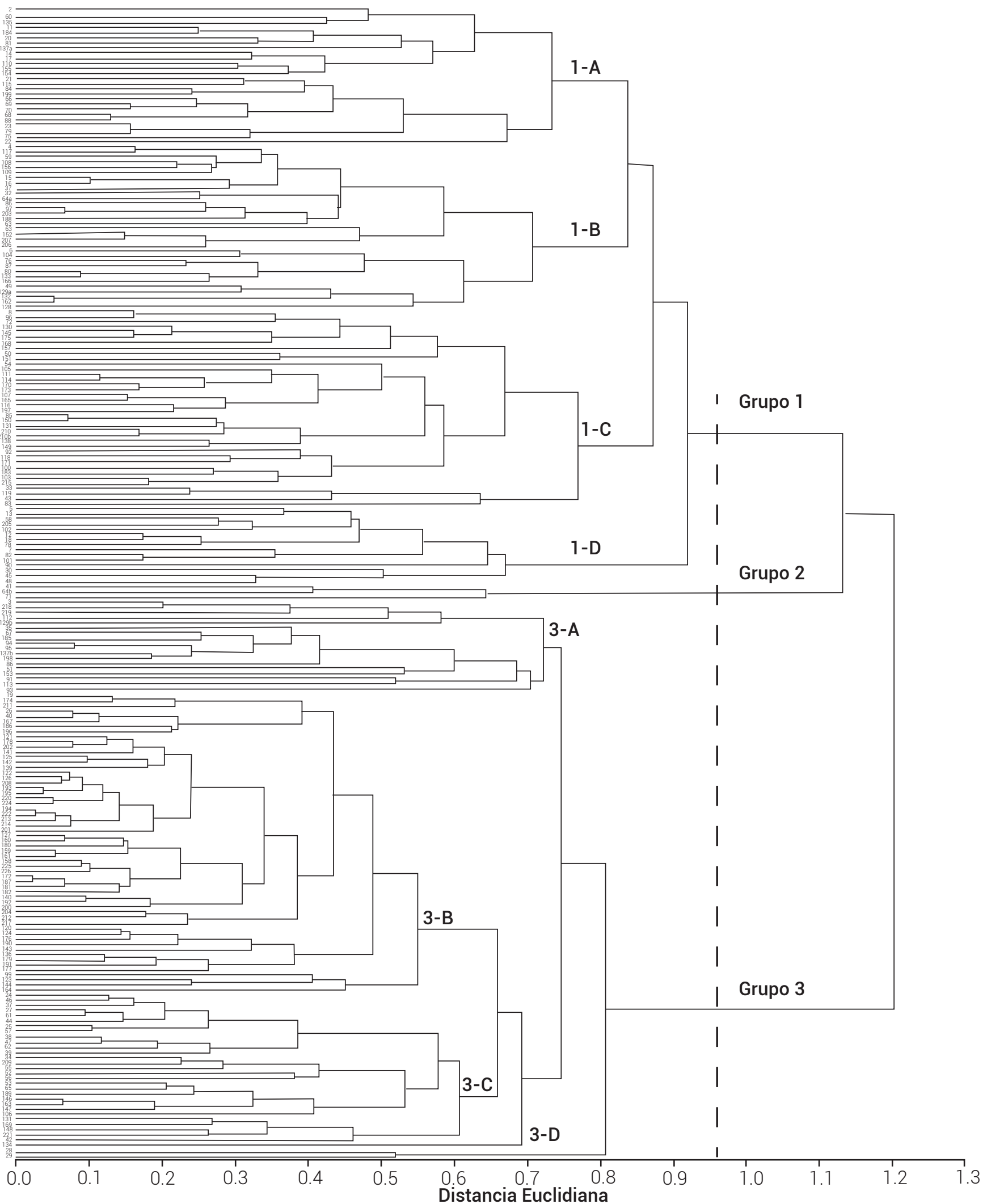

Figura 3. Dendrograma de 222 ejemplares de $C$. papaya generado con distancias euclidianas con base en 10 características morfológicas y el método de agrupamiento UPGMA. 
El Grupo 1 se formó por 111 ejemplares que provienen de huertos familiares, y que se distribuyeron en cuatro subgrupos con similitud en el diámetro de cavidad central (Cuadro 2). Los frutos presentaron colores de pulpa de verde amarilla (5GY9/12) a roja (10R6/16). El Subgrupo 1-A exhibió frutos en forma de pera, con una relación longitud/ diámetro de 2.4; el 1-B, frutos en forma de pera, pero con los valores altos (2.6) en la relación longitud/diámetro y la longitud $(29.4 \mathrm{~cm})$; el 1-C mostró valores intermedios tanto en las dimensiones como en la forma, al ser elíptica, con 1.7 de relación longitud/diámetro; en el 1-D, la forma del fruto fue globular, alcanzó el mayor diámetro $(12.7 \mathrm{~cm})$ y 1.8 de relación longitud/diámetro.

El Grupo 2 estuvo integrado por tres recolectas de papayo, con valor intermedio de longitud del fruto (Cuadro 2). Se distinguió de los Grupos 1 y 3 por mostrar mayor relación longitud/diámetro (3.4) y grosor de pulpa $(3.2 \mathrm{~cm})$, cavidad ovárica pequeña (2.0 cm de diámetro) y forma oblonga-aperada. Los colores de pulpa fueron de 2.5Y8/10 a 7.5YR7/12 (de amarilla a amarilla-anaranjada).

El Grupo 3 incluyó 108 recolectas subdivididas en cuatro subgrupos que mostraron semejanza en la longitud y diámetro del fruto con $6.6 \mathrm{~cm}$ y $4.5 \mathrm{~cm}$, respectivamente (Cuadro 2). El color de pulpa de 5GY9/12 a 7.5Y9/8 (de verde-amarilla a amarilla) fue más frecuente en comparación con la amarilla-anaranjada (7.5YR7/12). Dentro de este grupo, 76 \% de los ejemplares se localizaron en varios tipos de vegetación, en los que destaca la selva alta perennifolia, aunque algunos de ellos se situaron en los acahuales (Helianthus spp.) y el $24 \%$ se encontró en huertos familiares. Al respecto, Caballero (1992) señaló que es posible encontrar este tipo de poblaciones en los huertos, ya que sus frutos contribuyen a la alimentación familiar. También Chávez-Pesqueira y Núñez-Farfán (2016) encontraron germoplasma silvestre en la vegetación de acahuales. En este Grupo 3, el dendrograma no logró diferenciar, dentro de tipos silvestres, los recolectados en vegetación natural de aquellos de huertos familiares. Este resultado tiene coherencia con las observaciones de Coppens d'Eeckenbrugge et al. (2007), quienes mediante marcadores morfológicos e izoenzimáticos caracterizaron germoplasma silvestre proveniente de áreas naturales y de huertos familiares, y tampoco encontraron diferencias.

El Subgrupo 3-A presentó principalmente frutos de forma elíptica al mostrar 1.7 de relación longitud/ diámetro, con cavidad central tipo angular; el 3-B presentó valores intermedios (1.5) de relación longitud/diámetro, correspondiente a la forma redonda; el 3-C y 3-D mostraron forma oval al tener 1.2 de relación longitud/diámetro, pero ambos discreparon en la forma del extremo peduncular y prominencia de aristas.
Badillo (1971) y Manshardt y Zee (1994) encontraron en germoplasma silvestre frutos con longitud de 2 a $10 \mathrm{~cm}$ y 1.5 a $6 \mathrm{~cm}$ de diámetro, de forma redonda, pulpa amarilla - amarilla-anaranjada, pulpa escasa y cavidad central reducida; características similares a las encontradas en el Grupo 3 en el presente estudio.

\section{CONCLUSIONES}

La variabilidad morfológica de los ejemplares de $C$. papaya nativos de México descritos muestra importantes diferencias en la forma, dimensiones y tipo del extremo peduncular en fruto. Estas características del fruto son las que más contribuyen a la variabilidad morfológica entre el germoplasma evaluado; sin embargo, con base en las dimensiones del fruto se detectó un patrón gradual que podría estar asociado con el proceso de domesticación. Esto revela revela que los pobladores de las diferentes regiones probablemente se enfocaron en la obtención de frutos grandes con mucha pulpa comestible y diversidad de colores de pulpa. Paralelamente se pudo haber favorecido la presencia de plantas hermafroditas que no están presentes en las poblaciones silvestres estudiadas. Se identificaron tres grupos con ejemplares de papayo fenotípicamente diferente entre ellos. Se observó una continuidad en dimensiones y forma del fruto entre y dentro de grupos definidos en el análisis de conglomerados.

\section{AGRADECIMIENTOS}

Al Dr. Catarino Ávila Reséndiz ${ }^{\dagger}$ por liderar este trabajo. Al CONACYT por la beca otorgada para estudios de postgrado del primer autor. AI SINAREFI-SAGARPA por el financiamiento económico. A los Drs. Elías Hernández Castro, Francisco Palemón Alberto, Pedro Cisneros Saguilán, Lilia Alcaraz Meléndez, Gustavo Sosa Pérez; M.C. José Luis Hernández; Biól. Janeth Jobo, que ayudaron en la recolecta de $C$. papaya. A los propietarios de los huertos familiares por donar dicho material.

\section{BIBLIOGRAFÍA}

Aikpokpodion P. 0. (2012) Assessment of genetic diversity in horticultural and morphological traits among papaya (Carica papaya) accessions in Nigeria. Fruits 67:173-187, https://doi. org/10.1051/fruits/2012011

Allendorf F. W., G. H. Luikart and S. N. Aitken (2012) Conservation and the Genetics of Populations. Second edition. Wiley-Blackwell. Chichester, UK. 624 p.

Ara N., M. Moniruzzaman, F. Begum, M. Moniruzzaman and R. Khatoon (2016) Genetic divergence analysis in papaya (Carica papaya L.) genotypes. Bangladesh Journal of Agricultural Research 41:647-656, https://doi.org/10.3329/bjar.v41i4.30697

Asudi G. O., F. K. Ombwara, F. K. Rimberia, A. B. Nyende, E. M. Ateka, L. S. Wamocho, D. Shitanda and A. Onyango (2010) Morphological diversity of Kenyan papaya germplasm. African Journal of Biotechnology 9:8754-8762. 
Badillo V. M. (1971) Monografía de la Familia Caricaceae. Asociación de Profesores. Universidad Central de Venezuela. Maracay, Venezuela. $221 \mathrm{p}$.

Caballero J. (1992) Maya homegardens: past, present and future. Etnoecológica 1:35-54

Castellen M. S., C. A. S. Ledo, E. J. Oliverira, L. S. M. Filho e J. L. Dantas L. (2007) Caracterização de acessos do banco ativo de germoplasma de mamão por meio de análise multivariada. Magistra 19:299-303.

Chan Y. K. and S. C. Ooi (1975) Preliminary studies on papaya selection in Malaysia. Malaysian Agricultural Journal 50:67-77.

Chávez-Pesqueira M. and J. Núñez-Farfán (2016) Genetic diversity and structure of wild populations of Carica papaya in Northern Mesoamerica inferred by nuclear microsatellites and chloroplast markers. Annals of Botany 118:1293-1306, https:// doi.org/10.1093/aob/mcw183

Coppens d'Eeckenbrugge G., M. T. Restrepo, D. Jiménez and E. Mora (2007) Morphological and isozyme characterization of common papaya in Costa Rica. Acta Horticulturae 740:109-120, https:// doi.org/10.17660/ActaHortic.2007.740.11

Da Silva F. F., M. G. Pereira, H. C. C. Ramos, P. C. D. Junior, T. N. S. Pereira, A. P. Viana, R. F. Daher and G. A. Ferreguetti (2008) Estimation of genetic parameters related to morpho-agronomic and fruit quality traits of papaya. Crop Breeding and Applied Biotechnology 8:65-73.

Dantas J. L. L., R. M. S. Pinto, J. F. Lima e F. R. Ferreira (2000) Catálogo de Germoplasma de Mamão (Carica papaya L.). Documentos No. 94. Embrapa Mandioca e Fruticultura. Cruz das Almas, Bahia Brasil. $40 \mathrm{p}$

Fuentes G. and J. M. Santamaría (2014) Papaya (Carica papaya L.): origin, domestication, and production. In: Genetics and Genomics of Papaya. Ming and P. H. Moore (eds.). Springer-Verlag. New York. pp:3-15

Gabriel K. R. (1971) The biplot graphic display of matrices with application to principal component analysis. Biometrika 58:453-467, https://doi.org/10.1093/biomet/58.3.453

IBPGR, International Board for Plant Genetic Resources (1986) Genetic Resources of Tropical and Sub-tropical Fruits and Nuts (Excluding Musa). International Board for Plant Genetic Resources. Rome, Italy. $162 \mathrm{p}$.

Jiménez M. A. (2011) Herbolaria Mexicana. Biblioteca Básica de Agricultura. Colegio de Postgraduados. Texcoco, Estado de México. $531 p$

Manshardt R. M. and F. T. P. Zee (1994) Papaya germplasm and breeding in Hawaii. Fruit Varieties Journal 48:146-152.

Manshardt R. (2014) History and future of the Solo papaya. In: Genetics and Genomics of Papaya. R. Ming and P. H. Moore (eds.) Springer-Verlag. New York. pp:95-113

Moore P. H. (2014) Phenotypic and genetic diversity of papaya. In Genetics and Genomics of Papaya. R. Ming and P. H. Moore (eds.). Springer-Verlag. New York. pp:35-45.

Munsell Color (Firm) (1977) Munsell ${ }^{\circledR}$ Color Charts for Plant Tissues. Second edition. Macbeth Division of Kollmorgen Instruments Corporation. New Windsor, New York. $6 \mathrm{p}$

Nakasone H. Y. and R. E. Paull (1998) Tropical Fruits. CAB International. Wallingford, UK. $445 \mathrm{p}$.

Oliveira E. J., D. S. Lima, R. S. Lucena, T. B. N. Motta and J. L. L. Dantas (2010) Correlações genéticas e análise de trilha para número de frutos comerciais por planta em mamoeiro. Pesquisa Agropecuária Brasileira 45:855-862, https://doi.org/10.1590/S0100 204X2010000800011

Oliveira E. J., N. L. P. Dias and J. L. L. Dantas (2012) Selection of morphoagronomic descriptors for characterization of papaya cultivars. Euphytica 185:253-265, https://doi.org/10.1007/s10681-0110565-0

Pla L. E. (1986) Análisis Multivariado: Método de Componentes Principales. Programa Regional de Desarrollo Científico y Tecnológico. Organización de Estados Americanos. Washington D. C. 94 p.

Rawlings J. 0., S. G. Pantula and D. A. Dickey (1988) Applied Regression Analysis: A Research Tool. Second edition. Springer. New York. $659 \mathrm{p}$

SAS Institute (2004) SAS/STAT 9.1 User's Guide. SAS Institute Inc. Cary, USA. $5121 \mathrm{p}$

Singh K. and A. Kumar (2010) Genetic variability and correlation studies in papaya under Bihar conditions. Acta Horticulturae 851:145150, https://doi.org/10.17660/ActaHortic. 2010.851.20

Sompak S., R. Siriyan, T. Nimkingrat, S. Chaikiattiyos and U. Khumcha (2014) Papaya (Carica papaya) germplasm collection at Si Sa Ket Horticultural Research Center. Acta Horticulturae 1022:63-68, https://doi.org/10.17660/ActaHortic.2014.1022.6

Soriano-Melgar L. L. A., L. Alcaraz-Meléndez, M. Rodríguez-Álvarez y S. RealCosío (2016) Colecta y conservación in vitro y ex situ de recursos fitogenéticos de Carica papaya L. Agroproductividad 9:28-32.

UPOV, International Union for the Protection of New Varieties of Plants (2008) Papaya. UPOV Code: CARIC_PAP Carica papaya L. Guidelines for the conduct of tests for distinctness, uniformity and stability. International Union for the Protection of New Varieties of Plants. Geneva, Switzerland. $29 \mathrm{p}$

Vázquez C. M., M. J. Zavala L., F. A. Contreras M., F. Espadas G., A. Navarrete Y., L. F. Sánchez T. and J. M. Santamaría (2014) New cultivars derived from crosses between commercial cultivar and a wild population of papaya rescued at its center of origin. Journal of Botany 2014, Article ID 829354, https://doi.org/10.1155/2014/829354

Wall M. M. and S. Tripathi (2014) Papaya nutritional analysis. In: Genetics and Genomics of Papaya. R. Ming and P. H. Moore (eds.) Springer-Verlag. New York. pp:377-390 
